\title{
From cellular function to global impact: the vascular perspective on COVID-19
}

\author{
Shira A. Strauss, MD \\ Chanhee Seo, BSc \\ Marc Carrier, MD, MSc \\ Prasad Jetty, MD, MSc
}

Accepted April 7, 2021

\author{
Correspondence to: \\ SA Strauss \\ 606-131 Holland Avenue \\ Ottawa, ON K1Y 3A2 \\ shira.strauss@medportal.ca
}

DOI: $10.1503 /$ cjs. 023820
Since COVID-19 was declared a pandemic a year ago, our understanding of its effects on the vascular system has slowly evolved. At the cellular level, SARSCoV-2 - the virus that causes COVID-19 - accesses the vascular endothelium through the angiotensin-converting enzyme 2 (ACE-2) receptor and induces proinflammatory and prothrombotic responses. At the clinical level, these pathways lead to thromboembolic events that affect the pulmonary, extracranial, mesenteric, and lower extremity vessels. At the population level, the presence of vascular risk factors predisposes individuals to more severe forms of COVID-19, whereas the absence of vascular risk factors does not spare patients with COVID-19 from unprecedented rates of stroke, pulmonary embolism and acute limb ischemia. Finally, at the community and global level, the fear of COVID-19, measures taken to limit the spread of SARS-CoV-2 and reallocation of limited hospital resources have led to delayed presentations of severe forms of ischemia, surgery cancellations and missed opportunities for limb salvage. The purpose of this narrative review is to present some of the data on COVID-19, from cellular mechanisms to clinical manifestations, and discuss its impact on the local and global surgical communities from a vascular perspective.

Depuis que la COVID-19 s'est vu donner le statut de pandémie il y a 1 an, notre connaissance des effets de cette maladie sur le système vasculaire a évolué. À l'échelle cellulaire, le SRAS-CoV-2 - le virus qui cause la COVID-19 - accède à l'endothélium vasculaire par le récepteur de l'enzyme de conversion de l'angiotensine-2 (ACE-2) et provoque des réponses proinflammatoires et prothrombotiques. À l'échelle clinique, ces réponses peuvent mener à une activité thromboembolique touchant les vaisseaux pulmonaires, extracrâniens, mésentériques et des membres inférieurs. À l'échelle populationnelle, la présence chez certaines personnes de facteurs de risque vasculaires les prédispose à une forme plus grave de la COVID-19, mais l'absence de ces facteurs n'empêche pas les patients atteints de la COVID-19 de présenter des taux sans précédent d'AVC, d'embolie pulmonaire et d'ischémie aiguë aux membres. Enfin, à l'échelle locale et mondiale, la peur entourant la COVID-19, les mesures prises pour en endiguer la propagation et le redéploiement des ressources limitées des hôpitaux ont mené au report de visites à l'hôpital pour des formes graves d'ischémie, à l'annulation de chirurgies et à des occasions manquées de préserver des membres. La présente revue non systématique a pour objectif de présenter une partie des données sur la COVID-19, de ses mécanismes cellulaires à ses manifestations cliniques, et de discuter des répercussions de la crise sur les communautés chirurgicales locales et mondiales, dans une optique vasculaire.

$\mathbf{F}$ ew have been exempt from the effects of the COVID-19 pandemic. In March 2020, our outpatient clinics and elective surgeries were cancelled almost overnight as we were told to brace ourselves for a biologic tsunami. Over the ensuing few months, surgeons were forced to triage patients on their operating room (OR) wait lists and advocate for their sickest, while some were redeployed to COVID-19 wards and testing centres.

Since the arrival of presumed patient zero to North America in January $2020,{ }^{1}$ our evolving understanding of the implications of this virus remains limited. The variability in susceptibility and response of each individual cell, patient and community infected with SARS-CoV-2 - the virus that causes COVID-19 - reflects the heterogeneity in viral genetics, patients, community demographics, environmental factors and access to testing and 
health resources. In addition, our knowledge of COVID19 has depended on the rapid global dissemination of information, often without the usual rigour of scientific scrutiny. ${ }^{2,3}$ Case reports, case series and even cohort studies are susceptible to some imprecision in capturing the true incidence of complications, considering the likely small fraction of SARS-CoV-2 infections confirmed with polymerase chain reaction (PCR) testing compared with the true incidence in the general population.

Notwithstanding this uncertainty, the continuous appraisal and reappraisal of incoming data will allow us to better understand the changing expressions on the face of COVID-19. Although it has revealed diverse involvement of respiratory, gastrointestinal, neurologic, integumentary and cardiovascular systems, the association of COVID-19 with vascular endothelium may be a common biologic thread that weaves this disease through different organs. ${ }^{4}$

This review presents some of the data on COVID-19, from cellular activity to clinical manifestations, and discusses its effect on the surgical and global community from a vascular perspective.

\section{Covid-19 AT the Cellular LeVel}

\section{COVID-19 and the alveolus}

When SARS-CoV-2 enters the respiratory system through the nose or mouth, spike proteins found on the surface of the virus recognize and preferentially bind to angiotensin-converting enzyme 2 (ACE-2) receptors on type II alveolar cells. Type II alveolar cells are responsible for secreting surfactant and preventing alveoli from collapsing. ${ }^{5}$ From there, the virus hijacks the host cell's machinery to replicate and travel to adjacent cells. This process destroys the host cell and causes it to enter pyroptosis, a highly inflammatory pathway of cell death induced by pathogen invasion. ${ }^{6}$

Oxygen exchange is subsequently impeded in several ways. First, dysfunctional type II alveolar cells result in less surfactant, with ensuing alveolar collapse. The immune response also destroys adjacent type I alveolar cells, which are the main site of oxygen exchange. Furthermore, the inflammatory cascade causes fluid buildup in the alveoli, which may lead to further alveolar collapse, pneumonia, and possible progression to acute respiratory distress syndrome (ARDS; see a thorough diagram analysis at www.azuravesta.com/covid-19-pandemic). ${ }^{5}$ Finally, the ensuing inflammatory cascade interacts with the coagulation pathway to induce prothrombotic effects and complications such as pulmonary embolism (PE). ${ }^{6}$

\section{COVID-19 and the ACE-2 receptor}

Beyond its respiratory effects, COVID-19 both interacts with additional organ systems (as evidenced by its gastrointestinal [GI] manifestations) and disproportionately affects those with baseline multiorgan disease. ${ }^{7}$ Closer examination of the ACE-2 receptor, the target entry portal for SARS-CoV-2, may help account for the multisystem impact of COVID-19. ACE-2 is a membrane-associated aminopeptidase expressed throughout the human body, including in the lungs, kidneys, brain, GI tract and cardiovascular system. ${ }^{8} \mathrm{It}$ acts as a negative regulator of ACE in the reninangiotensin-aldosterone system (RAAS), converting angiotensin (Ang) II into Ang (1-7) and leading to downstream vasodilatory, antiapoptotic and antiproliferative effects. ${ }^{9}$ By occupying the ACE- 2 receptor, SARS-CoV-2 leads to increased levels of Ang II and consequently, vasoconstriction, inflammation, cell proliferation and enhanced thrombosis. ${ }^{10,11}$ Researchers have hypothesized that conditions associated with chronically imbalanced ACE/ACE-2 and Ang II levels, such as hypertension, may exacerbate this mechanism and account for the increased risk of severe disease in comorbid patients. ${ }^{11}$ Indeed, endothelial dysfunction is increasingly recognized as a fundamental component of COVID-19 pathophysiology. ${ }^{4,11}$

\section{COVID-19 and the vascular endothelium}

SARS-CoV-2 can access the circulatory system via ACE-2 receptors in the vascular endothelium and trigger a similar inflammatory reaction as seen in the pulmonary system. The activated vascular endothelium upregulates the expression of von Willebrand factor and adhesion molecules. This leads to recruitment of neutrophils, platelets and complement to the site of activity. The neutrophils release neutrophil extracellular traps (NETs), which activate the intrinsic pathway of the clotting cascade. Meanwhile, complement upregulates the expression of tissue factor, which activates the extrinsic pathway. Together, the intrinsic and extrinsic pathways lead to unlimited thrombin generation and, ultimately, fibrin clot formation and thrombus propagation throughout the vasculature. ${ }^{6}$

Accordingly, it follows that patients with COVID-19 are at increased risk for thromboembolic events. Virchow's triad for venous thromboembolism (VTE) - blood stasis, hypercoagulability and endothelial injury - often coexists in patients who are most vulnerable to severe COVID-19. When acute illness strikes, comorbid patients may become bedridden for extended periods and quickly deteriorate into sepsis. Active inflammation and endothelial dysfunction contribute to hemostatic abnormalities characteristic of the underlying prothrombotic state, including elevated D-dimer levels and fibrin degradation products and prolonged prothrombin time. Finally, arterial and venous thromboembolic events, and even disseminated intravascular coagulopathy (DIC), may follow. ${ }^{12}$ 


\section{COVID-19 AT THE PATIENT LEVEL: CLINICAL} MANIFESTATIONS

\section{Venous thromboembolism}

Cardiovascular complications among patients with severe COVID-19 are not only highly prevalent, but also associated with a more than 5-fold increased risk of mortality. ${ }^{13,14}$ Studies have consistently shown that VTE, and especially $\mathrm{PE}$, accounts for the largest proportion of cardiovascular thromboembolic events. ${ }^{13-16}$ The incidence of VTE among patients with COVID-19 ranges from $0 \%$ to $69 \%,{ }^{17-19}$ according to a Swiss systematic review of VTE data from 1369 patients with COVID-19 across 11 studies. ${ }^{19}$ The studies varied in size, type (observational v. experimental and prospective v. retrospective), use of antithrombotic therapy, method of diagnosis (use of systematic screening and type of imaging), definition of VTE (deep vein thrombosis [DVT], PE, or both), and severity of illness (intensive care unit [ICU] v. ward patients). ${ }^{19}$ These differences help account for the discrepancy between the $0 \%$ VTE incidence seen in the 16-person study by Ranucci and colleagues, ${ }^{17}$ in which $100 \%$ of participants received intermediate dosing antithrombotic therapy and none underwent VTE screening, and the $69 \%$ incidence in the ICU study by Llitjos and colleagues, ${ }^{18}$ in which full-leg ultrasonography VTE screening was performed twice in the first week of follow up. An additional 6 of the 11 studies included in the systematic review were restricted to ICU inpatients, and participants in all but 1 of these received pharmacological thromboprophylaxis (at either prophylactic, intermediate, or therapeutic doses). Nevertheless, VTE rates remained above $9 \%$ in each of these studies. ${ }^{19}$ Among the 7 studies that reported rates of both $\mathrm{PE}$ and DVT, PE was the more common VTE manifestation in 6 studies. Only 1 study, with frequent full-leg screening ultrasonography, reported a higher rate of DVT (78\% DVT v. $23 \%$ PE). ${ }^{18}$

Two autopsy case series conducted in Germany and Austria revealed that VTE is common in patients with COVID-19, even when not suspected clinically. ${ }^{20,21}$ In the former study, computed tomography (CT) scans and full autopsies were routinely performed on the first 12 consecutive deaths from PCR-confirmed COVID-19 in Hamburg, regardless of the presumed cause of death. This study identified massive PE, derived from lower extremity DVT, as the cause of death in $30 \%$ of patients and confirmed the presence of DVT in $58 \% .{ }^{20}$ In the latter study, directed autopsies were conducted in 12 patients with COVID-19 who were not clinically suspected of having VTE. Strikingly, this case series reported the presence of $\mathrm{PE}$ in $100 \%$ of patients, with clear histological evidence of both thrombotic and embolic occlusions in pulmonary arteries of various sizes. ${ }^{21}$
To illustrate the elevated thrombotic risk in patients with COVID-19, 2 studies examined VTE rates in patients with COVID-19 compared with non-COVID-19 counterparts. ${ }^{15,22}$ The first compared a cohort of 107 patients with COVID-19 admitted to the ICU with pneumonia to a historical control of 196 non-COVID-19 patients who were admitted with pneumonia during the same period 1 year prior. The study found a significantly higher frequency of PE in patients with COVID-19 than in the historical controls $(20.6 \%$ v. $6.1 \%)$, which remained consistent when comparing the patients with COVID-19 to a subcohort of patients with influenza pneumonia of similar disease severity $(20.6 \%$ v. $7.5 \%){ }^{22}$ The second study compared a cohort of 150 patients with COVID-19 admitted to the ICU with ARDS to a historical control of 233 non-COVID-19 bacterial/viral ARDS patients. Similarly, the study found that the patients with COVID-19 ARDS had significantly more thrombotic complications ( $11.7 \%$ v. $4.8 \%)$, specifically more PEs ( $11.7 \%$ v. $2.1 \%)$, than their matched non-COVID-19 counterparts. ${ }^{15}$

\section{Disseminated intravascular coagulopathy in COVID-19}

DIC describes a system of dysfunctional coagulation and fibrinolysis that can lead to overt thrombosis and/or hemorrhage. DIC is both a clinical and laboratory diagnosis that requires a high index of suspicion in the appropriate clinical setting. ${ }^{23}$ The International Society on Thrombosis and Hemostasis (ISTH) scoring system, which allots points based on abnormalities in platelet count, fibrinogen level, prothrombin time and D-dimer, can be used to identify such patients, with scores of 5 or higher being compatible with overt DIC (Table 1). ${ }^{24}$

\begin{tabular}{|c|c|}
\hline Parameter & Score* \\
\hline \multicolumn{2}{|l|}{ Platelet count } \\
\hline$\geq 100000$ & 0 \\
\hline 50000-99999 & 1 \\
\hline$\leq 50000$ & 2 \\
\hline \multicolumn{2}{|l|}{ Fibrinogen level } \\
\hline$\geq 1 \mathrm{~g} / \mathrm{L}$ & 0 \\
\hline$<1 \mathrm{~g} / \mathrm{L}$ & 1 \\
\hline \multicolumn{2}{|c|}{ Prothrombin time prolongation above ULN, s } \\
\hline$<3$ & 0 \\
\hline $3-6$ & 1 \\
\hline$>6$ & 2 \\
\hline \multicolumn{2}{|l|}{ D-dimer } \\
\hline$<2 \times$ ULN & 0 \\
\hline $2-4 \times$ ULN & 2 \\
\hline$>4 \times$ ULN & 3 \\
\hline \multicolumn{2}{|c|}{$\begin{array}{l}\text { DIC = disseminated intravascular coagulopathy; ISTH = International Society on } \\
\text { Thrombosis and Hemostasis; ULN = upper limit of normal. }\end{array}$} \\
\hline${ }^{*}$ Scores $\geq 5$ points ar & \\
\hline
\end{tabular}


Considering the prothrombotic nature of COVID-19, practitioners have suspected an association between SARS$\mathrm{CoV}-2$ and DIC and considered how this might affect a patient's prognosis. Indeed, a study on coagulation parameters conducted in 183 COVID-19 inpatients with pneumonia revealed that mortality in this cohort was associated with higher $\mathrm{D}$-dimer and fibrin degradation product levels as well as prolonged prothrombin time (PT) and activated partial thromboplastin time (aPTT). Furthermore, nonsurvivors met criteria for DIC more frequently than survivors $(71.4 \%$ v. $0.6 \%){ }^{25}$ This study suggested that the development of DIC in patients with COVID-19 may be a predictor of adverse outcomes. However, as noted in a recent review on COVID-19 and thromboembolic disease, these findings were inconsistent. ${ }^{12}$ Mild thrombocytopenia and elevated D-dimer levels were fairly common among patients with COVID-19 and were occasionally associated with adverse events such as ICU admission and death, but the comparison of coagulation parameters across 11 studies in patients with COVID-19 did not reveal a consistent association between individual laboratory abnormalities (such as aPTT, PT, and international normalized ratio) and severity of illness. ${ }^{12}$

\section{Prophylaxis and treatment of VTE and DIC}

Study variability and rapidly emerging guidelines have generated uncertainty for clinicians trying to follow treatment recommendations. The aforementioned clinical review, ${ }^{12}$ published in the fournal of the American College of Cardiology in June 2020, was a collaborative effort endorsed by multiple thrombosis, vascular and cardiology societies, including the International Society on Thrombosis and Haemostasis, North American Throm- bosis Forum, European Society of Vascular Medicine, International Union of Angiology and European Society of Cardiology Working Group on Pulmonary Circulation and Right Ventricular Function. Based on a thorough literature review and Delphi exercise, a set of consensus guidelines on the prophylaxis and treatment of VTE in patients with COVID-19 was developed.12 Beyond the usual VTE prophylaxis, the authors felt the data were insufficient to recommend intermediate or therapeutic anticoagulation in patients with mild, moderate or severe infections without thrombotic complications. There was also insufficient evidence to support routine screening for VTE in this population, except to inform the need for antithrombotic prophylaxis after discharge in patients with moderate to severe COVID-19 and suspected or confirmed DIC (Table 2). ${ }^{12}$

For patients with COVID-19 who present with VTE or an acute coronary syndrome (ACS), the authors recommended anticoagulation and guideline-directed medical therapy as per the ACS algorithm, respectively. The decision to proceed with more invasive treatments, such as catheter-directed therapy, angiography and percutaneous coronary intervention, or surgery, should involve careful consideration of the COVID-19 risk facing the patient and health care team balanced with the risk posed by the suspected or confirmed VTE/ACS. In high-risk COVID-19 patients, health care providers should consider systemic fibrinolysis as an alternative to more invasive therapies if warranted. ${ }^{12}$

\section{Cardiac events in COVID-19}

COVID-19 is associated with cardiac injury, but not exclusively through prothrombotic pathways. In a retro-

Table 2. Highlights from the Consensus Recommendation on Antithrombotic Therapy for prophylaxis in patients with mild to severe COVID-19 with and without DIC ${ }^{7}$

\begin{tabular}{|c|c|}
\hline COVID-19 severity & Recommendations \\
\hline Mild COVID-19 (outpatient) & $\begin{array}{ll}\text { - } & \text { Encourage ambulation } \\
\text { - } & \text { Consider DVTp if increased VTE risk and minimal bleeding risk } \\
\text { - } & \text { Continue antithrombotic agents if already prescribed for prior known thrombotic disease }\end{array}$ \\
\hline $\begin{array}{l}\text { Moderate to severe COVID-19 without DIC } \\
\text { (hospitalized) }\end{array}$ & $\begin{array}{l}\text { - } \quad \text { DVTp should be administered to prevent VTE } \\
\text { - } \quad \text { Consider intermittent pneumatic compressions if anti-thrombotics contraindicated } \\
\text { Insufficient data to support therapeutic or intermediate-dose anticoagulation or routine screening for } \\
\text { VTE }\end{array}$ \\
\hline $\begin{array}{l}\text { Moderate to severe COVID-19 and suspected or } \\
\text { confirmed DIC and no overt bleeding (hospital- } \\
\text { ized) }\end{array}$ & $\begin{array}{l}\text { - } \\
\text { - InTufficient data to support therapeutic or intermediate-dose anticoagulation } \\
\text { - If on chronic therapeutic anticoagulation, reduce intensity of anticoagulation unless very high risk of } \\
\text { thrombosis } \\
\text { - If indication for DAPT, continue DAPT if plt }>50 \text { 000; reduce to MAPT if plt } 25000-50000 \text {; discontinue } \\
\text { if plt }<25000 \text { (however must individualize decision) } \\
\text { - If inpatient with COVID-19 now being discharged, routine VTE screening is reasonable to guide need for } \\
\text { DVTp for up to } 45 \text { days postdischarge; consider DVTp if high risk for thrombotic events } \\
\text { - Encourage ambulation }\end{array}$ \\
\hline
\end{tabular}


spective cohort study of 416 patients admitted to hospital with COVID-19, cardiac injury - defined as a troponin elevation above the 99th percentile with or without ECG or echocardiographic changes occurred in $19.7 \%$ of patients during their hospital stay. Notably, not only was cardiac injury a predictor of inhospital mortality (hazard ratio [HR] 4.25), but it was also associated with a myriad of complications, including ARDS, acute kidney injury, electrolyte disturbances and coagulation disorders. ${ }^{26}$ Recently, Hendren and colleagues ${ }^{27}$ postulated a spectrum of mechanisms to account for the exceedingly high rates of cardiac injury associated with COVID-19.27 Most of these cardiac injuries occurred in the absence of coronary artery thrombosis and were in fact attributed to myocarditis, cytokine dysregulation, stress cardiomyopathy, arrhythmias (made worse in the setting of hypoxia) and heart failure (often related to $\mathrm{PE}$ ). The mechanisms of injury are not fully understood, but may be related to ACE2-mediated direct damage, hypoxia-induced injury, microvascular damage (related to angiospasm, vessel hyperpermeability and dysfunctional coagulation) and the systemic inflammatory response. ${ }^{27}$

\section{Peripheral arterial thromboembolism and COVID-19}

Although arterial complications are less frequently reported with COVID-19 than venous complications, ${ }^{13-16}$ the peripheral arterial system has been targeted by SARSCoV-2 infection. In a cohort study of 1419 inpatients with COVID-19 in Spain, only 14 patients (1\%) developed systemic arterial thrombotic events, but the mortality rate among those with an arterial event was $28.6 \%{ }^{28}$ Arterial thromboembolic disease is not only highly deadly in patients with COVID-19, but also less predictable than the usual atherosclerotic variety. These features are especially pronounced among COVID-19 patients with ischemic strokes. ${ }^{29}$ Ischemic stroke among inpatients with COVID-19 has an incidence of $0.05 \%-5.7 \%,{ }^{14,28,30}$ affecting young patients as well as old, with and without typical risk factors. ${ }^{29,31}$ In a recent case series of 5 patients with COVID-19 who presented with stroke over a 2-week period, all were younger than 50 years, had few to no atherosclerotic risk factors, and their National Institutes of Health Stroke Scale scores were consistent with severe strokes (mean score 17). In contrast, over the previous 12 months, an average of only 0.73 patients younger than 50 years presented with large-vessel stroke over any given 2-week period. ${ }^{32}$ A subsequent retrospective study of 20 COVID-19 patients with acute ischemic stroke as their presenting symptom found that large vessel occlusion was present in $75 \%$. Among those with large vessel occlusions, most were age 50 or younger, and $60 \%$ had no typical cardiovascular risk factors. ${ }^{31}$ Finally, a systematic review examining stroke risk, characteristics and out- comes in patients with COVID-19 corroborated the aforementioned findings: among 160 patients admitted with stroke and COVID-19, the median age was 65 years, with 29 patients (18\%) younger than 50 years. Among younger patients with ischemic stroke, $50 \%$ had no preceding symptoms of COVID-19, 69\% had large vessel occlusions, and $50 \%$ either died or remained hospitalized by the end of the study. ${ }^{29}$ Strokes associated with COVID-19 are a unique variety, with their poor predictability and adverse outcomes likely stemming from the hypercoagulable and proinflammatory state that is inherent to the virus.

Information about thromboembolic disease in the aorta, visceral vessels and lower extremity arteries of patients with COVID-19 is slowly emerging, but remains largely limited to case reports and small case series..$^{33-37}$ Many of these reports document cases of acute limb ischemia (ALI) in patients with COVID-19 with no history of peripheral arterial disease (PAD). ${ }^{33-35}$ In 1 report, a marked spike in D-dimer levels - which led to same-day initiation of intermediate enoxaparin therapy - was the sole warning sign for an extensive below-knee arterial thrombosis that developed 1 day later. ${ }^{33}$ The systemic inflammatory cytokine storm and coagulation abnormalities associated with COVID-19 were proposed as likely predisposing these otherwise low-risk individuals to ALI. ${ }^{34,35}$ Pathology was supportive, demonstrating a combination of occlusive thrombosis, endothelial proliferation and highly inflammatory infiltration in 1 series. ${ }^{35}$ Unfortunately, these changes can affect the entire arterial tree, as evidenced by a 56-year-old patient with COVID-19 who presented initially with an acute ischemic stroke and subsequently developed free floating aortic thrombus, superior mesenteric artery (SMA) occlusion and associated bowel ischemia necessitating endovascular thrombectomy and an extensive bowel resection. ${ }^{36}$

In 2 of the COVID-19 epicentres - Lombardy, Italy and New York, USA - small studies reported on ALI during the pandemic. ${ }^{38,39}$ The Italian study identified 20 COVID-19 patients with ALI and noted a significant increase in the overall cumulative incidence of ALI during the pandemic compared with the same time period the previous year (23 of 141 vascular emergencies v. 3 of 163 ). Among COVID-19 patients with ALI who underwent an attempt at revascularization (17 of 20), success - defined as the absence of 30-day reocclusion, 24-hour persistent foot ischemia and 24-hour major amputation or death was lower than expected $(70.6 \%)$, and mortality was $40 \%{ }^{38}$ In the New York study, 10 patients with COVID19 and ALI had even worse outcomes, with a $50 \%$ success rate among those who underwent revascularization and a $40 \%$ overall 30-day mortality. ${ }^{39}$ While each of these studies has helped advance our understanding of the relationship between COVID-19, the arterial system and patient outcomes, there is still much to improve upon. 
Fortunately, collaborative initiatives to examine the effects of COVID-19 on aortic dissection and to quantify the impact of vascular surgical care delays due to the pandemic, are currently underway. ${ }^{40,41}$

\section{COVID chilblains ("COVID toes")}

There have been several media reports of patients with COVID-19 presenting with purpuric lesions to the toes, resembling chilblains or pernio. ${ }^{42,43}$ Chilblains is a known inflammatory condition characterized by cold-induced skin changes in predisposed individuals. The etiology may be idiopathic or associated with systemic disease, including autoimmune disorders, genetic mutations, hematologic malignancy and infection (e.g., Epstein-Barr virus). The first case of chilblains associated with COVID-19 was reported in a 13 year-old in Italy in March 2020. Over the following months, additional reports of similar presentations amassed, leading to the formation of COVID-19 registries of skin manifestations by dermatology and podiatry groups. ${ }^{44}$ In the context of COVID-19, the mechanism of chilblains is not yet entirely understood, but the phenomenon seems to be more common in younger individuals with milder infection. In some cases patients actually test negative for the virus, which may reflect low viral loads or an alternate etiology entirely. ${ }^{45}$ Histopathology from skin biopsies of the feet and toes reveals a combination of lymphocytic vasculitis and inflammation, thrombosis, and endothelial damage, as well as SARS-CoV-2 viral particles within the endothelial cells. ${ }^{46}$ To date, there are no reports of macrovascular ischemic injury leading to this presentation in patients with COVID-19. Current management of "COVID toes" is generally expectant, with most resolving after a few weeks. Some clinicians have considered the role of antithrombotics or calcium channel blockers, but there is minimal evidence to support their use at this time. ${ }^{47}$

\section{COVID-19 AT THE COMMUNITY LEVEL: IMPACT ON VASCULAR PATIENTS AND PRACTITIONERS}

\section{Vascular patients with COVID-19}

While large-scale epidemiological studies on patients with COVID-19 rarely examine the prevalence of PAD in this population, they frequently assess the prevalence of PAD risk factors. An early study of 1100 patients with COVID-19 in China illustrated that patients with a history of smoking, diabetes, hypertension, chronic obstructive pulmonary disease and coronary artery disease tended to have more severe COVID-19 and worse outcomes. ${ }^{7}$ Likewise, a large study from China's Infectious Disease Information System followed more than 72000 patients with confirmed or suspected COVID-19 and found that hypertension, dyslipidemia and cardiovascular disease were more common in those who died from COVID-19 than in the overall cohort of confirmed cases. ${ }^{48}$ From these data, it can be inferred that vascular patients with COVID-19, who have an exceptionally high prevalence of these risk factors, are among those with the highest risk for severe disease and associated adverse events.

\section{Vascular patients without COVID-19}

Even when vascular patients do not have COVID-19, it often affects them. Amidst lockdowns, closure of outpatient activities, fear of contracting SARS-CoV-2 from the hospital and limited access to the OR, vascular patients have experienced delays in the assessment and management of their often time-sensitive conditions. ${ }^{49}$ Although triaging will always occur on an individual patient basis in relation to hospital resources, decision algorithms and priority classifications require further exploration. For some conditions, there are sufficient data to guide treatment within specific timeframes to prevent worse outcomes; e.g., ALI (within hours) or a transient ischemic attack from severe carotid stenosis $(14 \mathrm{~d}) .^{50,51}$ However, for conditions such as chronic limb threatening ischemia (CLTI) or abdominal aortic aneurysms (AAAs), the impact of delayed care is more difficult to define. In 1 Italian institution, vascular surgeons observed that the number of amputations performed in patients with PAD in March-April 2020 was nearly 50\% higher than in the same time period the previous year. ${ }^{49}$ Similarly, at a hospital in Beijing, fewer PAD surgeries were performed, severity of presenting ischemia was worse, and perioperative complications were higher in January-March 2020 than in the same time period a year prior. ${ }^{52}$ These studies suggest that the impact of COVID-19 on the health care system may be leading to worse outcomes for patients with PAD, whose chances for limb salvage decrease as their disease progresses. Monitoring outpatients during the pandemic is challenging; however, vascular surgeons must remain particularly vigilant in their follow up and find innovative ways to accurately assess, triage and advocate for their patients.

\section{The Ottawa Experience}

The Ottawa Hospital (TOH) Civic Campus is the only centre providing vascular surgery to the 1.3 million residents living in the Ottawa and broader Champlain Local Health Integration Network. When the pandemic was declared in March 2020, our institution responded to the pressure on resources similarly to other hospitals worldwide. Vascular outpatient clinics and academic activities became virtual, except for a single weekly inperson urgent care clinic. Elective OR time substantially 


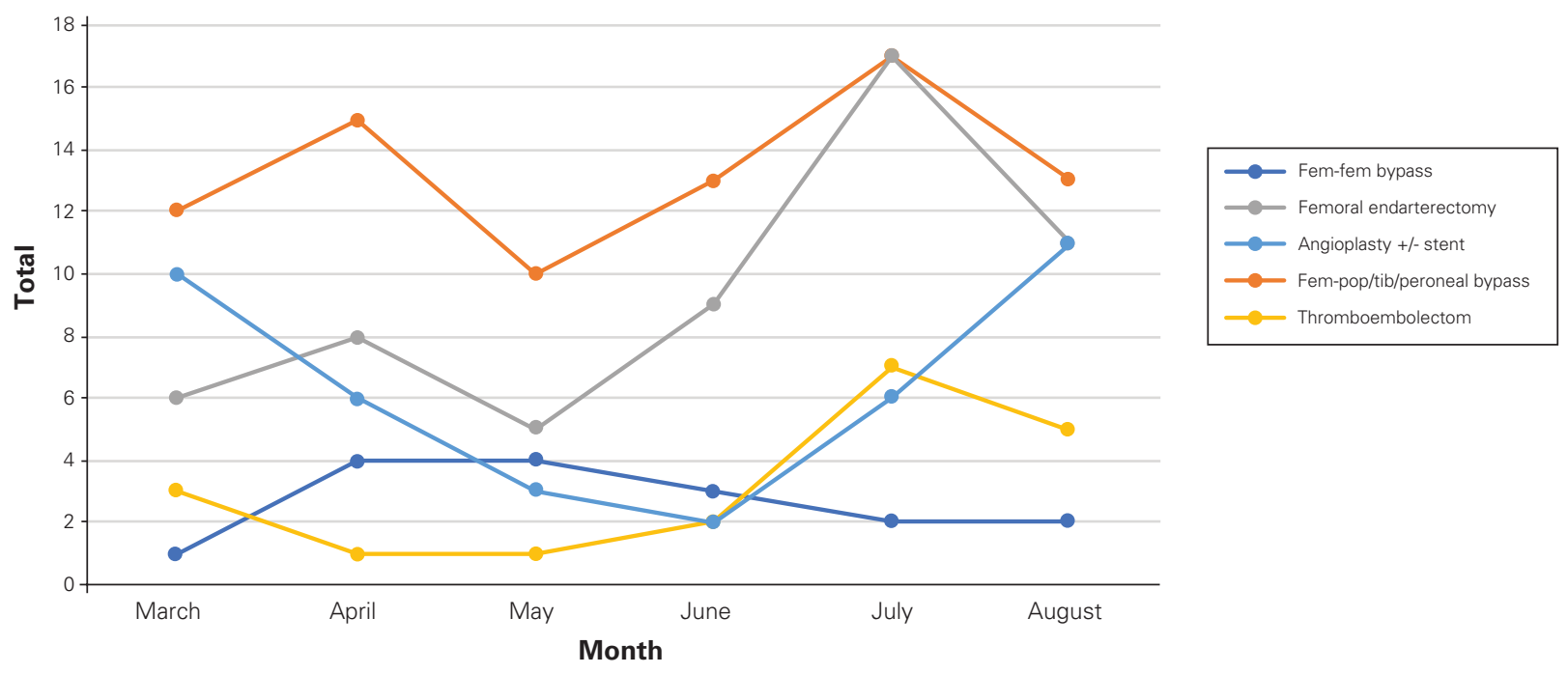

Figure 1. Trends in infrainguinal limb salvage procedures from March to August, 2020, at The Ottawa Hospital Civic Campus. Femfem = femorofemoral; fem-pop = femoral politeal; tib = femoral-tibial.

decreased, and cases were triaged. Vascular staff were reduced to the minimum number required in hospital, and all extraneous team members were sent home after morning rounds (both to facilitate physical distancing and to preserve the health of the team in case staff or residents were redeployed or became ill).

From March to August 2020, only 243 of 398 elective vascular surgeries were performed, leaving a backlog of 155 cases. However, this number substantially underestimates the true backlog of elective surgeries, since only urgent cases - such as CLTI, large AAAs, and symptomatic carotid stenosis - were permitted to fill the elective time. Similarly, it is challenging to accurately assess differences between the number of emergent surgeries during March-August 2020 (221) compared with the same time period the previous year (250). Since the pandemic was declared, surgical trends primarily reflected logistical limitations on the OR rather than reluctance to attempt limb salvage due to delayed and advanced presentations of ischemia: common revascularization procedures slowed when OR restrictions were at their peak in May and steadily increased as ORs reopened at the beginning of the summer (Figure 1). Major and minor amputations followed a similar trend. The slower resumption of endovascular procedures (angioplasty and stenting) in the OR attests to our collaborative efforts with interventional radiology (IR), which enabled endovascular procedures to continue in the IR suite in an expedited fashion and often in an outpatient capacity rather than using inpatient beds and limited OR resources.
Our centre did not experience much difference in the number of infrainguinal bypasses or major (i.e., above ankle) amputations in April-August 2020 compared with the same period the previous year $(68 \mathrm{v}$. 60 infrainguinal bypasses; 31 v. 36 major amputations). Our centre's ability to successfully manage urgent limb salvage procedures during the pandemic reflects multiple factors. First, elective OR time was triaged on a departmental level against the caseloads of other surgical specialties and, because of the urgency of many vascular procedures, vascular surgery was often allotted proportionally more OR time than other subspecialties. Second, the division of vascular surgery already functioned as a group practice before the pandemic, allowing for triaging and pooling of cases performed on the basis of urgency and surgeon availability on a week-to-week basis. Finally, our centre was fortunate not to experience as overwhelming an influx of patients with COVID-19 as initially anticipated: from Mar. 1 to Oct. 31, 2020, TOH Civic Campus admitted a total of 69 patients with confirmed COVID-19. Nonetheless, the negative effect of delays as a result of the OR shutdowns during the pandemic remains to be determined as the backlog of elective cases is addressed.

\section{COVID-19 AT THE GLOBAL LEVEL: THE VASCULAR REACTION AND FUTURE DIRECTIONS}

Globally, vascular surgeons have taken similar measures to prepare for the COVID-19 surge and manage limited 
health care resources. In March 2020, Chris Imray, President of the Vascular Society for Great Britain and Ireland, issued a set of recommendations to help vascular surgeons navigate these difficult decisions..$^{53}$ General principles included reducing unnecessary exposure to hospitals, deferring less urgent or elective cases and proceeding with urgent ones, shifting to virtual platforms where possible, and considering resource usage when selecting course of treatment (e.g., consider endovascular rather than open approach if it may prevent an ICU stay). Vascular surgeons in Singapore shared their experience with the pandemic to date, reporting many of the tactics mentioned above, as well as suspended overseas travel and conference leave, strict use of masks and physical distancing, initiation of a delivery service for chronic medications, and use of local/regional anesthesia to limit ventilator use and aerosolization. ${ }^{54}$

We cannot yet appreciate the full impact of COVID-19, nor our efforts to defend against it. Many patients remain on surgical waiting lists owing to the lockdowns during the first and second waves, and the various pandemic waves will likely have ripple effects for months, and possibly years, to come. While it can be difficult to stay appraised of the rapidly evolving information, the variability in the literature will help us understand nuances in the way that COVID-19 affects different patients and populations. As a specialty, vascular surgeons have evolved during the pandemic as well, pivoting to virtual clinic platforms, piloting COVID-19 vascular initiatives (e.g., dedicated line service ${ }^{55}$ ), and gaining a better appreciation of the impact of delayed care on our patient population. COVID-19 has profound effects on the vascular system, patients, practitioners and global community; the more we understand, the better we can respond at each of these levels.

Affiliations: From the Division of Vascular Surgery, University of Ottawa at The Ottawa Hospital, Ottawa, Ont. (Strauss, Jetty); the Faculty of Medicine, University of Ottawa, Ottawa, Ont. (Strauss, Seo, Carrier, Jetty); the Division of Hematology, Department of Medicine, University of Ottawa, The Ottawa Hospital, Ottawa, Ont. (Carrier); and the Ottawa Hospital Research Institute, Ottawa, Ont. (Carrier).

Competing interests: Marc Carrier reports research support outside the submitted work from Leo Pharma and Bristol-Myers Squibb and honoraria from Sanofi Aventis, Pfizer, Leo Pharma and Bayer. He is an advisory board member of Sanofi Aventis, Leo Pharma and Valeo Pharma. No other competing interests were declared.

Contributors: S. Strauss deigned the study. C. Seo acquired the data, which M. Carrier and P. Jetty analyzed. S. Strauss and P. Jetty wrote the article, which all authors reviewed. All authors gave final approval of the version to be published.

Content licence: This is an Open Access article distributed in accordance with the terms of the Creative Commons Attribution (CC BYNC-ND 4.0) licence, which permits use, distribution and reproduction in any medium, provided that the original publication is properly cited, the use is noncommercial (i.e., research or educational use), and no modifications or adaptations are made. See: https://creativecommons. org/licenses/by-nc-nd/4.0/

\section{References}

1. First North American case of coronavirus in Washington State. Global News 2020 January 21. Available: https://globalnews.ca/video/6444973/ first-north-american-case-of-coronavirus-in-washington-state/ (accessed 2020 Oct. 20).

2. Tingley K. Coronavirus is forcing medical research to speed up. The New York Times 2020 May 21. Available: https://www.nytimes. com/2020/04/21/magazine/coronavirus-scientific-journals-research. html (accessed 2020 Oct. 20).

3. Fast pace of scientific publishing on COVID comes with problems. Inside Higher Ed 2020 June 8. Available: https://www.insidehighered. com/news/2020/06/08/fast-pace-scientific-publishing-covid-comes -problems (accessed 2020 Oct. 20).

4. Libby P, Lüscher T. COVID-19 is, in the end, an endothelial disease. Eur Heart 7 2020;41:3038-44.

5. The science behind COVID-19. Azuravesta Design [infographic]. Available: https://www.azuravesta.com/covid-19-pandemic (accessed 2020 Oct. 20).

6. McFadyen JD. Stevens Hannah, Peter Karlheinz. The emerging threat of (micro)thrombosis in COVID-19 and its therapeutic implications. Circ Res 2020;127:571-87.

7. Guan W-J, Ni Z-Y, Hu Y, et al. Clinical characteristics of coronavirus disease 2019 in China. N Engl 7 Med 2020;382:1708-20.

8. Hamming I, Timens W, Bulthuis MLC, et al. Tissue distribution of ACE2 protein, the functional receptor for SARS coronavirus. A first step in understanding SARS pathogenesis. 7 Pathol 2004;203:631-7.

9. Pagliaro P, Penna C. ACE/ACE2 ratio: a key also in 2019 coronavirus disease (COVID-19)? Front Med (Lausanne) 2020;7:335.

10. Jin Y, Ji W, Yang H, et al. Endothelial activation and dysfunction in COVID-19: from basic mechanisms to potential therapeutic approaches. Signal Transduct Target Ther 2020;5:293.

11. Gan R, Rosoman NP, Henshaw DJE, et al. COVID-19 as a viral functional ACE2 deficiency disorder with ACE2 related multi-organ disease. Med Hypotheses 2020;144:110024.

12. Bikdeli B, Madhavan MV, Jimenez D, et al. COVID-19 and Thrombotic or thromboembolic disease: implications for prevention, antithrombotic therapy, and follow-up: JACC state-of-the-art review. $\mathcal{F}$ Am Coll Cardiol 2020;75:2950-73.

13. Klok FA, Kruip MJHA, van der Meer NJM, et al. Incidence of thrombotic complications in critically ill ICU patients with COVID19. Thromb Res 2020;191:145-7.

14. Klok FA, Kruip MJHA, van der Meer NJM, et al. Confirmation of the high cumulative incidence of thrombotic complications in critically ill ICU patients with COVID-19: an updated analysis. Thromb Res 2020;191:148-50.

15. Helms J, Tacquard C, Severac F, et al. High risk of thrombosis in patients with severe SARS-CoV-2 infection: a multicenter prospective cohort study. Intensive Care Med 2020;46:1089-98.

16. Lodigiani C, Iapichino G, Carenzo L, et al. Venous and arterial thromboembolic complications in COVID-19 patients admitted to an academic hospital in Milan, Italy. Thromb Res 2020;191:9-14.

17. Ranucci M, Ballotta A, Dedda UD, et al. The procoagulant pattern of patients with COVID-19 acute respiratory distress syndrome. 7 Thromb Haemost 2020;18:1747-51.

18. Llitjos J-F, Leclerc M, Chochois C, et al. High incidence of venous thromboembolic events in anticoagulated severe COVID-19 patients. $\mathcal{F}$ Thromb Haemost 2020;18:1743-6.

19. Fontana P, Casini A, Robert-Ebadi H, et al. Venous thromboembolism in COVID-19: systematic review of reported risks and current guidelines. Swiss Med Wkly 2020;150:w20301.

20. Wichmann D, Sperhake J-P, Lütgehetmann M, et al. Autopsy findings and venous thromboembolism in patients with COVID-19. Ann Intern Med 2020;173:268-77. 
21. Lax SF, Skok K, Zechner P, et al. Pulmonary arterial thrombosis in covid-19 with fatal outcome: results from a prospective, singlecenter, clinicopathologic case series. Ann Intern Med 2020; 173:M20-2566.

22. Julien P, Julien G, Morgan C, et al. Pulmonary embolism in patients with COVID-19. Circulation 2020;142:184-6.

23. Disseminated intravascular coagulation (DIC) in adults: evaluation and management. UpToDate. Availalbe: https://www.uptodate.com/ contents/disseminated-intravascular-coagulation-dic-in-adults -evaluation-and-management? search=DIC\&source=search_result\& selected Title $=1 \sim 150 \&$ usage_type $=$ default $\&$ display_rank=1\#H12 (accessed 2020 Sept. 30).

24. Toh CH, Hoots WK. The scoring system of the Scientific and Standardisation Committee on Disseminated Intravascular Coagulation of the International Society on Thrombosis and Haemostasis: a 5-year overview. 7 Thromb Haemost 2007;5:604-6.

25. Tang N, Li D, Wang X, et al. Abnormal coagulation parameters are associated with poor prognosis in patients with novel coronavirus pneumonia. 7 Thromb Haemost 2020;18:844-7.

26. Shi S, Qin M, Shen B, et al. Association of cardiac injury with mortality in hospitalized patients with COVID-19 in Wuhan, China. 7AMA Cardiol 2020;5:802-10.

27. Hendren NS, Drazner MH, Bozkurt B, et al. Description and proposed management of the acute COVID-19 cardiovascular syndrome. Circulation 2020;141:1903-14.

28. Cantador E, Núñez A, Sobrino P, et al. Incidence and consequences of systemic arterial thrombotic events in COVID-19 patients. 7 Thromb Thrombolysis 2020; doi: 10.1007/s11239-020-02176-7.

29. Fridman S, Bullrich MB, Jimenez-Ruiz A, et al. Stroke risk, phenotypes, and death in COVID-19: systematic review and newly reported cases. Neurology 2020;95:e3373-85.

30. Mao L, Jin H, Wang M, et al. Neurologic manifestations of hospitalized patients with coronavirus disease 2019 in Wuhan, China. FAMA Neurol 2020;77:683.

31. John S, Kesav P, Mifsud VA, et al. Characteristics of large-vessel occlusion associated with COVID-19 and ischemic stroke. A7NR Am 7 Neuroradiol 2020;41:2263-8.

32. Oxley TJ, Mocco J, Majidi S, et al. Large-vessel stroke as a presenting feature of COVID-19 in the young. N Engl 7 Med 2020;382:e60.

33. Warrior K, Chung PA, Ahmed N, et al. Acute limb ischemia due to arterial thrombosis associated with coronavirus disease 2019. Crit Care Explor 2020;2:e140.

34. Perini P, Nabulsi B, Massoni CB, et al. Acute limb ischaemia in two young, non-atherosclerotic patients with COVID-19. Lancet 2020;395:1546.

35. Cañas EG, Gaibar AG, Lorenzo LR, et al. Acute peripheral arterial thrombosis in COVID-19. Role of endothelial inflammation. B7S Br 7 Surg 2020;107:e444-5.

36. Azouz E, Yang S, Monnier-Cholley L, et al. Systemic arterial thrombosis and acute mesenteric ischemia in a patient with COVID-19. Intensive Care Med 2020;46:1464-5.

37. Patel P, Yu Y, Zia S, et al. Systemic thrombolysis as initial treatment of COVID-19 associated acute aortoiliac and lower extremity arterial thrombosis. Ann Vasc Surg 2021;70:297-301.

38. Bellosta R, Luzzani L, Natalini G, et al. Acute limb ischemia in patients with COVID-19 pneumonia. 7 Vasc Surg 2020;72:1864-72.

39. Lewis CT, Dayal R, Sundaram V, et al. Revascularization outcomes in COVID-19 patients with acute limb ischemia. 7 Vasc Surg 2020;72:e374.

40. Seattle team secures funding to examine COVID-19 impacts on aortic dissection. Vascular Specialist 2020 July 28, 2020. Availalbe: https://vascularspecialistonline.com/seattle-team-secures-funding -to-examine-covid-19-impacts-on-aortic-dissection/ (accessed 2020 Oct. 22).

41. Mouawad NJ, Cuff RF, Hultgren R, et al. The Vascular Surgery COVID-19 Collaborative (VASCC). 7 Vasc Surg 2020;72:379-80.

42. Rabin RC. What is 'covid toe'? Maybe a strange sign of coronavirus infection. New York Times 2020 Sept. 11. Availalbe: https:// www.nytimes.com/2020/05/01/health/coronavirus-covid-toe.html (accessed 2020 Sept. 30).

43. New study finds evidence between COVID-19 and "COVID toes" symptom. CTV News 2020 Jul. 5. Availalbe: https://www.ctvnews. ca/health/coronavirus/new-study-finds-evidence-between-covid -19-and-covid-toes-symptom-1.5011460 (accessed 2020 Sept. 30)

44. Massey PR, Jones KM. Going viral: a brief history of chilblain-like skin lesions ("COVID toes") amidst the COVID-19 pandemic. Semin Oncol 2020;47:330-4.

45. Hernandez C, Bruckner AL. Focus on "COVID toes.". FAMA Dermatol 2020;156:1003.

46. Colmenero I, Santonja C, Alonso-Riaño M, et al. SARS-CoV-2 endothelial infection causes COVID-19 chilblains: histopathological, immunohistochemical and ultrastructural study of seven paediatric cases. Br 7 Dermatol 2020;183:729-37.

47. Cooke JP. How does COVID-19 cause cardiovascular disease? YouTube 2020 May 11. Available: https://www.youtube.com/ watch? $\mathrm{v}=\mathrm{aKb} \_$L1KaZ8A\&t=1054s (accessed 2020 Oct. 22).

48. Team TNCPERE. The epidemiological characteristics of an outbreak of 2019 novel coronavirus diseases (COVID-19) - China, 2020. China CDC Wkly 2020;2:113-22.

49. Sena G, Gallelli G. An increased severity of peripheral arterial disease in the COVID-19 era. 7 Vasc Surg 2020;72:758.

50. Aboyans V, Ricco J-B, Bartelink M-LEL, et al. Editor's Choice 2017 ESC guidelines on the diagnosis and treatment of peripheral arterial diseases, in collaboration with the European Society for Vascular Surgery (ESVS). Eur 7 Vasc Endovasc Surg 2018;55:305-68.

51. Brott TG, Halperin JL, Abbara S, et al. 2011 ASA/ACCF/AHA/ AANN/AANS/ACR/ASNR/CNS/SAIP/SCAI/SIR/SNIS/SVM/ SVS guideline on the management of patients with extracranial carotid and vertebral artery disease: executive summary. A report of the American College of Cardiology Foundation/American Heart Association Task Force on Practice Guidelines, and the American Stroke Association, American Association of Neuroscience Nurses, American Association of Neurological Surgeons, American College of Radiology, American Society of Neuroradiology, Congress of Neurological Surgeons, Society of Atherosclerosis Imaging and Prevention, Society for Cardiovascular Angiography and Interventions, Society of Interventional Radiology, Society of NeuroInterventional Surgery, Society for Vascular Medicine, and Society for Vascular Surgery. Circulation 2011; 124:489-532.

52. Li W, Chen X, Feng H. Impact of COVID-19 on peripheral arterial disease treatment. Ann Vasc Surg 2020;67:6-7.

53. Hudson J. Vascular Society issues guidance on COVID-19 and vascular surgery. Vascular News 2020 Mar. 24. Availalbe: https:// vascularnews.com/vascular-society-issues-guidance-on-covid -19-and-vascular-surgery/ (accessed 2020 Sept. 4).

54. Leong Tan GW, Chandrasekar S, Lo ZJ, et al. Early experience in the COVID-19 pandemic from a vascular surgery unit in a Singapore tertiary hospital. 7 Vasc Surg 2020;72:12-5.

55. O'Connor D, Wilderman M, Cao L, et al. Creation of a dedicated line service in the New Jersey epicenter of COVID-19. 7 Vasc Surg 2020;72:1159-60. 